\title{
CONTROLE QUÍMICO DE OVOS E NINFAS DE Bemisia tabaci BIÓTIPO B (HEMIPTERA: ALEYRODIDAE)
}

\author{
Giuliana Etore do Valle ${ }^{1,2 *}$; André Luiz Lourenção ${ }^{1,3} ;$ José Polese Soares Novo1 \\ ${ }^{1}$ Centro de Fitossanidade - IAC, C.P. 28 - CEP: 13001-970 - Campinas, SP. \\ ${ }^{2}$ Bolsista CAPES. \\ ${ }^{3}$ Bolsista CNPq. \\ *Autor correspondente <gevalle@yahoo.com.br>
}

\begin{abstract}
RESUMO: Moscas brancas são pragas de diversas culturas, causam redução na produção e na qualidade do produto. Inseticidas, com diferentes modos de ação vêm sendo utilizados, sem muito sucesso. Neste trabalho avaliou-se a eficiência de inseticidas sobre ovos de diferentes idades (1, 3 e 5 dias) e de ninfas de $1^{\circ}$ ínstar da mosca branca Bemisia tabaci biótipo B. Os experimentos foram realizados em condições de laboratório, sob temperatura de $25 \pm 2{ }^{\circ} \mathrm{C}$ e fotofase de 12 horas. Piriproxifem $\left(75 \mathrm{mg} \mathrm{L}^{-1}\right)$ atuou como excelente ovicida, sendo altamente eficiente, 3 dias de idade. Cartape $\left(500 \mathrm{mg} \mathrm{L}^{-1}\right)$ não teve efeito satisfatório, mas a $1000 \mathrm{mg}$ $\mathrm{L}^{-1}$ foi eficiente em ovos com até 5 dias de idade. Quanto à ação sobre ninfas de $1^{\circ}$ ínstar, para pulverizações na face superior das folhas, piriproxifem, a $75 \mathrm{mg} \mathrm{L}^{-1}$, apresentou eficiência na supressão da emergência de adultos, caracterizando presença de ação translaminar; buprofezim, cartape, acefato e fempiroximate não apresentaram eficiência no controle de ninfas. Quando aplicado na face inferior das folhas, buprofezim (375 $\mathrm{mg} \mathrm{L}^{-1}$ ) apresentou eficiência comparável à de piriproxifem, impedindo a emergência de adultos.

Palavras-chave: Bemisia argentifolii, Insecta, mosca branca, inseticidas
\end{abstract}

\section{CHEMICAL CONTROL OF Bemisia tabaci B BIOTYPE (HEMIPTERA: ALEYRODIDAE) EGGS AND NYMPHS}

\begin{abstract}
Whiteflies are pests of many crops, cause severe yield losses and reduce crop quality. Insecticides of different modes of action have been used without success. In this work, the efficiency of five insecticides on Bemisia tabaci B biotype (= B. argentifolii) eggs and nymphs was evaluated under laboratory conditions ( $\mathrm{T}=25 \pm 2^{\circ} \mathrm{C} ; 12 \mathrm{~h}$ photophase). In relation to the ovicidal effect, pyriproxyfen $\left(75 \mathrm{mg} \mathrm{L}^{-1}\right)$ caused egg hatching inhibition up to three day old eggs. Cartap showed efficiency only at the concentration of $1000 \mathrm{mg} \mathrm{L}^{-1}$, supressing the viability of eggs up to five days. Soybean leaves with first instar nymphs were treated in the upper surface with pyriproxyfen $\left(75 \mathrm{mg} \mathrm{L}^{-1}\right)$, buprofezin $\left(375 \mathrm{mg} \mathrm{L}^{-1}\right)$, cartap $\left(1000 \mathrm{mg} \mathrm{L}^{-1}\right)$, acephate $\left(750 \mathrm{mg} \mathrm{L}^{-1}\right)$ and fenpyroximate $\left(100 \mathrm{mg} \mathrm{L}^{-1}\right)$; in this condition, only pyriproxyfen suppressed adult emergence, indicating the presence of a translaminar effect. When leaves with nymphs were treated on both surfaces, buprofezin showed an efficiency similar to that of pyriproxyfen, also preventing adult emergence of the $B$. tabaci B biotype.

Key words: Bemisia argentifolii, Insecta, silverleaf whitefly, insecticides
\end{abstract}

\section{INTRODUÇÃO}

A mosca branca Bemisia tabaci biótipo B, descrita como B. argentifolii Bellows \& Perring (Bellows et al., 1994), é importante praga de culturas anuais, hortaliças e plantas ornamentais em praticamente todo o continente americano. No Brasil, a primeira constatação da presença desse inseto foi feita no Estado de São Paulo no início da década de 90 (Lourenção \& Nagai, 1994), quando surtos populacionais foram observados em plantas ornamentais (poinsétia e crisântemo) e em lavouras de abóbora (Cucurbita moschata) e de tomate (Lycopersicon esculentum), causando as desordens fisiológicas denominadas folha prateada da aboboreira e amadurecimento irregular dos frutos do tomateiro. Rapidamente nos anos seguintes, esse inseto atingiu as principais fronteiras agrícolas do país (França et al., 1996; Villas Bôas et al., 1997; Haji et al., 1997), sendo atualmente poucos os Estados onde ainda não ocorre. Além de induzir anomalias fisiológicas,
B. tabaci biótipo B transmite vírus (Brown et al., 1995), reduz o vigor da planta em virtude de se alimentar da seiva e introduzir substâncias tóxicas (Byrne \& Bellows, 1991) e também favorece a ocorrência de fumagina pela deposição de secreção açucarada, o que prejudica os processos fisiológicos da folha (Hendrix \& Wei, 1992), podendo, ainda, afetar a qualidade da produção quando incidir sobre frutos.

Para o desenvolvimento de estratégias de manejo dessa mosca branca, um dos pontos fundamentais é a disponibilidade de produtos eficientes e seletivos. Dessa forma, objetivou-se no presente trabalho, avaliar a eficiência de piriproxifem, análogo do hormônio juvenil, com atuação reconhecida sobre cochonilhas e moscas brancas (De Cock et al., 1995) e de buprofezim, regulador do crescimento de insetos que inibe a síntese de quitina em várias espécies de homópteros (De Cock et al., 1990), em comparação com outros inseticidas no controle de ovos e ninfas de $B$. tabaci biótipo B. 


\section{MATERIAL E MÉTODOS}

\section{Criação estoque de $B$. tabaci}

O trabalho foi realizado no período de maio a dezembro de 1998, no Núcleo Experimental de Campinas, localizado no Instituto Agronômico de Campinas (IAC). Os insetos utilizados no presente trabalho foram provenientes da criação de mosca branca do Setor de Entomologia do Centro de Fitossanidade do IAC. Essa criação vem sendo conduzida em soja, tomateiro e leiteiro (Euphorbia heterophylla), plantados em vasos e mantidos em condições de insetário, de dimensões de $3 \times 5 \mathrm{~m}$, com laterais de tela de malha fina e teto de vidro. Exemplares dessa criação foram identificados pela Dra. Judith K. Brown, Universidade do Arizona, como B. tabaci biótipo B.

\section{Ação letal em ovos de um dia}

Plantas de soja cv. Santa Rosa, no estágio de desenvolvimento vegetativo, foram colocadas nas bancadas do insetário de criação, sob alta pressão do inseto, para oviposição, a partir das $16 \mathrm{~h} 00$, sendo retiradas no dia seguinte às $8 \mathrm{~h} 00$. Foram examinadas folhas dessas plantas em microscópio estereoscópico, para separação e demarcação de uma área com dez ovos por folha. Para demarcação, utilizou-se caneta vermelha para retroprojetor, de ponta fina $(1,0 \mathrm{~mm})$. A seguir, cada folha com área demarcada foi retirada, submetida à pulverização, seca à sombra e individualizada em placa de Petri sob $25 \pm 2^{\circ} \mathrm{C}$ e fotofase de $12 \mathrm{~h}$, em condições de câmara climatizada (BOD). Os inseticidas foram aplicados nas folhas através de pulverizador de laboratório, a um volume de $184 \mathrm{~L} \mathrm{ha}^{-1}$, utilizando-se água destilada no preparo da calda (Tabela 1). Na testemunha aplicou-se somente água destilada. Inspecionaram-se diariamente as folhas para verificação da eclosão de ninfas.

Embora nas condições em que o experimento foi conduzido $\left(25 \pm 2^{\circ} \mathrm{C}\right.$ de temperatura e 12 horas de fotofase) e, o processo de eclosão de ninfas de $B$. tabaci demore de seis a oito dias (Butler Jr. et al., 1983; Villas Bôas et al., 1997), esperou-se dezenove dias para o encerramento do experimento, tempo suficiente para garantir que não haveria mais eclosões.

Tabela 1 - Ingredientes ativos utilizados nos experimentos de avaliação sobre ovos e ninfas de Bemisia tabaci biótipo B.

\begin{tabular}{lc}
\hline \multicolumn{1}{c}{ Ingrediente ativo } & Dose I. A. \\
\hline & $\mathrm{mg} \mathrm{L}^{-1}$ \\
buprofezim & 375 \\
piriproxifem & 75 \\
piriproxifem & 150 \\
acefato & 750 \\
fempiroximate & 100 \\
cartape & 500 \\
cartape & 1000 \\
\hline
\end{tabular}

Scientia Agricola, v.59, n.2, p.291-294, abr./jun. 2002
O delineamento utilizado foi o inteiramente casualizado, com sete tratamentos e dez repetições. Cada parcela constou de uma folha com dez ovos. Os dados, transformados em arc sen raiz $(x / 100)$ foram submetidos à análise de variância e as médias comparadas pelo teste de Tukey a $5 \%$.

\section{Ação letal em ovos de 1, 3 e 5 dias de idade}

Plantas de soja cv. Santa Rosa, no estágio de desenvolvimento vegetativo, foram expostas a adultos da mosca branca de forma idêntica a anterior. Folhas com áreas demarcadas com dez ovos foram pulverizadas com piriproxifem $\left(75 \mathrm{mg} \mathrm{L}^{-1}\right)$ e cartape $\left(1000 \mathrm{mg} \mathrm{L}^{-1}\right)$ e a testemunha com água. Após tratadas, as folhas foram secas à sombra, individualizadas em placas de Petri e mantidas em câmara climatizada (BOD) nas mesmas condições do teste anterior. As folhas destinadas à aplicação desses produtos em ovos de 3 e 5 dias foram também individualizadas e mantidas em BOD até essas datas, dois dias ou quatro dias após, para aplicação no mesmo horário. O volume utilizado foi o mesmo, ou seja, $184 \mathrm{~L} \mathrm{ha}^{-1}$. Inspecionaram-se diariamente as folhas, encerrando-se o experimento dezenove dias após a aplicação dos produtos.

O delineamento utilizado foi inteiramente casualizado, com parcelas subdivididas. As parcelas constaram dos tratamentos químicos e, as subparcelas, das três idades de ovos. Cada subparcela foi representada por uma folha com dez ovos, com dez repetições. As análises estatísticas foram idênticas às anteriores.

\section{Ação sobre ninfas de $1^{\circ}$ ínstar}

Neste teste, utilizaram-se copos plásticos com capacidade de $500 \mathrm{~mL}$, com duas plantas de soja cultivar IAC 19 em cada um. Quando as plantas apresentavam o primeiro trifólio completamente desenvolvido, foram infestadas durante 24 horas com adultos da mosca branca para oviposição. Após esse período, retiraram-se os adultos. Sete dias após, foi demarcada uma área com dez ninfas recém eclodidas em cada planta. Em seguida, foi efetuada a aplicação dos produtos (Tabela 1), pulverizados sobre a face superior das folhas, mediante uso de pulverizador de laboratório, a um volume de $184 \mathrm{~L} \mathrm{ha}^{-1}$, utilizando-se água destilada no preparo da calda. $\mathrm{Na}$ testemunha, aplicou-se somente água destilada. Diariamente acompanhou-se o desenvolvimento das ninfas até a emergência dos adultos.

Um segundo experimento foi conduzido de forma idêntica ao anterior, apenas modificando-se a forma de aplicação dos produtos, que foram pulverizados em ambas as faces das folhas. Os dois experimentos foram conduzidos em laboratório, em condições de fotoperíodo de 12 horas e temperatura de $25 \pm 2^{\circ} \mathrm{C}$. O delineamento utilizado em ambos foi inteiramente casualizado, com seis tratamentos e dez repetições. Cada parcela constou de uma planta com dez ninfas. Os procedimentos de análise foram semelhantes aos anteriores. 


\section{RESULTADOS E DISCUSSÃO}

Ação letal em ovos de um dia

Acefato, na dose de $750 \mathrm{mg} \mathrm{L}^{-1}$, e buprofezim, na dose de $375 \mathrm{mg} \mathrm{L}^{-1}$, não diferiram da testemunha (água) com relação ao efeito ovicida em $B$. tabaci biótipo B (Tabela 2). Houve alta percentagem de ninfas eclodidas nesses dois tratamentos, respectivamente 84 e $82 \%$, indicando que a fase de ovo não é afetada por esses produtos. Cartape, na dosagem de $500 \mathrm{mg} \mathrm{L}^{-1}$, apresentou comportamento intermediário, com $55 \%$ de ninfas eclodidas, e diferindo significativamente tanto de acefato e buprofezim como dos tratamentos mais eficientes, cartape a $1000 \mathrm{mg} \mathrm{L}^{-1}$, piriproxifem a 75 e a $150 \mathrm{mg} \mathrm{L}^{-1}$. O aumento na dose de cartape melhorou sua eficiência, praticamente impedindo a eclosão das ninfas e tendo desempenho comparável ao de piriproxifem. Este último produto, independentemente da dosagem, não permitiu a eclosão de ninfas, tendo $100 \%$ de eficiência sobre todos os ovos avaliados.

Resultados semelhantes com o uso de piriproxifem como ovicida para Trialeurodes vaporariorum, outra espécie de mosca branca, foram obtidos por De Cock et al. (1995) e Ishaaya et al. (1994), que verificaram a inibição da eclosão de ninfas em ovos de um dia, em proporções acima de $90 \%$. Para B. tabaci, Ishaaya \& Horowitz (1992) verificaram que a aplicação de piriproxifem em sistema de imersão de folhas com ovos de um dia proporcionou até $97 \%$ de inviabilidade.

Com relação a buprofezim, embora haja citação de que o tratamento de plantas de algodão com esse produto provoque redução na eclosão de ninfas (Ishaaya et al., 1988), não foram obtidos resultados similares no presente trabalho, mesmo tendo sido aplicada concentração de $375 \mathrm{mg} \mathrm{L}^{-1}$, três vezes superior à maior concentração usada por aqueles autores $\left(125 \mathrm{mg} \mathrm{L}^{-1}\right)$. Provavelmente, isto foi devido ao diferente método usado por Ishaaya et al. (1988), que trataram as plantas de algodão para posteriormente expô-las aos adultos, que foram afetados pelo produto e colocaram ovos com baixa viabilidade. No presente trabalho, aplicou-se buprofezim diretamente nos ovos, o que proporcionou respostas diferentes; infere-se, portanto, que esse produto tem melhor atuação na supressão da eclosão de ninfas quando em contato com os adultos.

\section{Ação letal em ovos de 1, 3 e 5 dias de idade}

A avaliação em ovos de um dia confirmou o efeito ovicida de cartape e piriproxifem, que não permitiu a eclosão de ninfas (Tabela 3). Já na aplicação feita em ovos de três dias, cartape, embora ainda com eficiente ação ovicida ( $82 \%$ de controle), diferiu significativamente do piriproxifem, que continuou apresentando $100 \%$ de controle. $\mathrm{Na}$ aplicação feita em ovos de cinco dias, muito próximos, portanto, da eclosão das ninfas, cartape manteve seu nível de controle ( $87 \%)$, semelhante àquele apresentado na aplicação em ovos de três dias, ao passo que piriproxifem não teve mais nenhum efeito ovicida e não diferiu da testemunha.

De forma semelhante, Ishaaya et al. (1994) observaram que ovos de $T$. vaporariorum com idade acima de três dias não têm a eclosão das ninfas afetada significativamente quando tratados com piriproxifem. Para a espécie B. tabaci, Ishaaya \& Horowitz (1992) constataram que ovos com 2-3 dias de idade tratados com piriproxifem foram menos suscetíveis que ovos de um dia e que o efeito de inibição da eclosão terminava em ovos com mais de quatro dias de idade. Os resultados aqui obtidos corroboram os dados disponíveis na literatura de que ovos mais velhos de mosca branca não são afetados pela aplicação de piriproxifem.

\section{Ação sobre ninfas de $1^{\circ}$ ínstar}

No teste em que se realizou a aplicação dos produtos na face superior das folhas, apenas piriproxifem, na dosagem de $75 \mathrm{mg} \mathrm{L}^{-1}$, apresentou eficiência na supressão de emergência de adultos da mosca branca, da ordem de $85 \%$ (Tabela 4). Os demais produtos não tiveram ação considerável sobre as ninfas, tendo os mais altos valores atingindo apenas $34 \%$ (buprofezim e acefato). Cartape e piriproxifem induziram índices de mortalidade de 17 e 16\%, respectivamente.

Tabela 2 - Percentagem de ninfas eclodidas de ovos de Bemisia tabaci biótipo $\mathrm{B}$, tratados no primeiro dia com quatro inseticidas, em condições de laboratório. Temperatura $25 \pm 2^{\circ} \mathrm{C}$; fotofase 12 horas $(n=10)$. Campinas, SP, 1998.

\begin{tabular}{|c|c|c|}
\hline Tratamento & Dose (I. A.) & Ninfas eclodidas* \\
\hline & ---- mg L'-1 ---- & ------------ \% - ----------- \\
\hline testemunha & & $93 a$ \\
\hline a cefato & 750 & $84 \mathrm{a}$ \\
\hline buprofezim & 375 & $82 \mathrm{a}$ \\
\hline cartape & 500 & $55 \mathrm{~b}$ \\
\hline cartape & 1000 & $4 \mathrm{c}$ \\
\hline piriproxifem & 75 & $0 \mathrm{c}$ \\
\hline piriproxifem & 150 & $0 \mathrm{c}$ \\
\hline C.V. (\%) & & 30,71 \\
\hline
\end{tabular}

${ }^{*}$ Médias seguidas pela mesma letra na coluna não diferem significativamente pelo Teste de Tukey a $5 \%$.

Tabela 3 - Percentagem de ninfas eclodidas de ovos de Bemisia tabaci biótipo $\mathrm{B}$, tratados em três idades diferentes, com dois inseticidas, em condições de laboratório. Temperatura $25 \pm 2^{\circ} \mathrm{C}$; fotofase 12 horas $(n=10)$. Campinas, SP, 1998.

\begin{tabular}{|c|c|c|c|c|}
\hline \multirow[t]{2}{*}{ Tratamento } & \multirow{2}{*}{$\begin{array}{c}\text { Dose (I.A.) } \\
\mathrm{mg} \mathrm{L}^{-1}\end{array}$} & \multicolumn{3}{|c|}{$\begin{array}{l}\text { Ninfas eclodidas } \\
\text { (\%) de ovos de }\end{array}$} \\
\hline & & 1 dia & 3 dias & 5 dias \\
\hline testemunha & & $94 \mathrm{a}$ & 94 a & $91 \mathrm{a}$ \\
\hline cartape & 1000 & $0 \mathrm{~b}$ & $18 \mathrm{~b}$ & $13 \mathrm{~b}$ \\
\hline piriproxifem & 75 & $0 \mathrm{~b}$ & $0 \mathrm{c}$ & 95 a \\
\hline C.V. tratamento (\%) & & & & 18,05 \\
\hline
\end{tabular}

C.V. idade (\%) 25,13

*Médias seguidas pela mesma letra na coluna não diferem pelo Teste de Tukey a $5 \%$. 
Tabela 4 - Inviabilidade de ninfas de $1^{\circ}$ ínstar de Bemisia tabaci biótipo B, tratadas com inseticidas apenas na face superior e nas duas faces. Temperatura $25 \pm 2^{\circ} \mathrm{C}$; fotofase 12 horas $(n=10)$. Campinas, SP, 1998.

\begin{tabular}{lccc}
\hline \multirow{2}{*}{ Tratamento } & \multirow{2}{*}{ Dose } & \multicolumn{2}{c}{ Inviabilidade ${ }^{*}$} \\
\cline { 3 - 4 } & & Aplicação na face superior & Aplicação em ambas as faces \\
\hline Piriproxifem & 75 & $85 \mathrm{a}$ & $85 \mathrm{a}$ \\
Buprofezim & 375 & $34 \mathrm{~b}$ & $83 \mathrm{a}$ \\
Cartape & 1000 & $17 \mathrm{bc}$ & $26 \mathrm{~b}$ \\
Acefato & 750 & $34 \mathrm{~b}$ & $20 \mathrm{~b}$ \\
Testemunha & & $7 \mathrm{c}$ & $15 \mathrm{~b}$ \\
Fenpiroximate & 100 & $16 \mathrm{bc}$ & $10 \mathrm{~b}$ \\
\hline Média & & 32,2 & 39,8 \\
\hline C.V. $(\%)$ & & 44,08 & 36,91 \\
\hline
\end{tabular}

*Médias seguidas pela mesma letra na coluna não diferem pelo Teste de Tukey a $5 \%$.

A eficiência de piriproxifem sobre ninfas de diferentes espécies de mosca branca tem sido relatada na literatura. Forte efeito de ação translaminar, mesmo em tratamentos com concentrações relativamente baixas do produto, foi observado em ninfas de B. tabaci (Ishaaya \& Horowitz, 1992). Esses autores observaram que, ninfas de $2^{\circ}$ e $3^{\circ}$ ínstares, tratadas com $5 \mathrm{mg} \mathrm{L}^{-1}$, tiveram pupação similar a testemunha, mas não houve emergência de adultos. Posteriormente, Horowitz \& Ishaaya (1994) confirmaram essas observações para essa mesma espécie, demonstrando que a aplicação de piriproxifem na face superior das folhas afeta as ninfas, suprimindo a emergência de adultos. Ishaaya et al. (1994) verificaram o mesmo para a espécie $T$. vaporariorum e Liu \& Stansly (1997) para B. argentifolii. No presente trabalho, também observou-se que não houve efeito direto de piriproxifem nas ninfas, mas sim na supressão de emergência de adultos quando são tratadas ninfas de $1^{\circ}$ ínstar.

No segundo experimento, em que os inseticidas foram aplicados em ambas as superfícies foliares, verificou-se que buprofezim apresentou eficiência comparável à do piriproxifem, reduzindo a emergência de adultos em $83 \%$ (Tabela 4). Os demais tratamentos, mesmo com pulverização direcionada à face inferior onde as ninfas estavam presentes, não tiveram melhorada sua eficiência se comparados com o ensaio anterior. Fica, portanto, caracterizado o buprofezim como não tendo ação translaminar, característica presente no piriproxifem conforme se infere comparando-se os resultados dos dois experimentos. A eficiência do buprofezim no controle de $B$. tabaci foi registrada por Gerling \& Sinai (1994). Mediante mergulho de folhas de algodão com ninfas do inseto em solução com esse inseticida, observaram que elas morriam durante passagens de ínstares, sendo que a sensibilidade era maior em ninfas de $1^{\circ}$ e $2^{\circ}$ ínstares.

O modo de ação do buprofezim difere do piriproxifem. Enquanto este último aparentemente não provoca grandes modificações externamente na ninfa, o buprofezim mata a ninfa logo no primeiro ínstar.

\section{REFERÊNCIAS BIBLIOGRÁFICAS}

BELLOWS JR., T.S.; PERRING, T.M.; GILL, R.J.; HEADRICK, D.H. Description of a species of Bemisia (Homoptera: Aleyrodidae). Annals of the Entomological Society of America, v.87, p.195-206, 1994.

BROWN, J.K.; COATS, S.A.; BEDFORD, I.D.; MARKHAM, P.G.; BIRD, J.; FROHLICH, D.R. Characterization and distribution of esterase electromorphs in the whitefly, Bemisia tabaci (Genn.) (Homoptera: Aleyrodidae). Biochemical Genetics, v.33, p.205-214, 1995.

BUTLER JR., G.D.; HENNEBERRY, T.J.; CLAYTON, T.E. Bemisia tabaci (Homoptera: Aleyrodidae): development, oviposition and longevity in relation to temperature. Annals of the Entomological Society of America, v.76, p.310-313, 1983.

BYRNE, D.N.; BELLOWS, T.S. Whitefly biology. Annual Review of Entomology, v.36, p.431-457, 1991.

DE COCK, A.; ISHAAYA, I.; DEGHEELE, D.; VEIEROV, D. Vapor toxicity and concentration-dependent persistence of buprofezin applied to cotton foliage for controlling the sweetpotato whitefly (Homoptera: Aleyrodidae). Journal of Economic Entomology, v.83, p.1254-1260, 1990.

DE COCK, A.; ISHAAYA, I.; VAN DE VEIRE, M.; DEGHEELE, D. Response of buprofezin-suscetible and-resistant strains of Trialeurodes vaporariorum (Homoptera: Aleyrodidae) to pyriproxyfen and diafenthiuron. Journal of Economic Entomology, v.88, p.763-767, 1995.

FRANÇA, F.H.; VILLAS BÔAS, G.L.V.; BRANCO, M.C. Ocorrência de Bemisia argentifolii Bellows \& Perring (Homoptera: Aleyrodidae) no Distrito Federal. Anais da Sociedade Entomológica do Brasil, v.25, p.369-372, 1996.

GERLING, D.; SINAI, P. Buprofezin effects on two parasitoid species of whitefly (Homoptera: Aleyrodidae). Journal of Economic Entomology, v.87, p.842-846, 1994.

HAJI, F.N.P.; LIMA, M.F.; ALENCAR, J.A. Histórico sobre mosca branca no Brasil. In: TALLER LATINOAMERICANO Y DEL CARIBE SOBRE MOSCAS BLANCAS Y GEMINIVIRUS, 6., Santo Domingo, 1997. Resumos. Santo Domingo: Junta Agroempresarial Dominicana Inc., 1997. p.5-8.

HENDRIX, D.L.; WEI, Y. Detection and elimination of honeydew excreted by the sweetpotato whitefly feeding upon cotton. In: BELTWIDE COTTON PRODUCTION CONFERENCE, Memphis, 1992. Proceedings. Memphis: National Cotton Council, 1992. p.671-673.

HOROWITZ, A.R.; ISHAAYA, I. Managing resistance to insect growth regulators in the sweetpotato whitefly (Homoptera: Aleyrodidae). Journal of Economic Entomology, v.87, p.866-871, 1994.

ISHAAYA, I.; HOROWITZ, A.R. Novel phenoxy juvenile hormone analog (pyriproxifen) supresses embryogenesis and adult emergence of sweetpotato whitefly (Homoptera: Aleyrodidae). Journal of Economic Entomology, v.85, p.2113-2117, 1992.

ISHAAYA, I.; MENDELSON, Z.; MELAMED-MADJAR, V. Effect of buprofezin on embryogenesis and progeny formation of sweetpotato whitefly (Homoptera: Aleyrodidae). Journal of Economic Entomology, v.81, p.781-784, 1988.

ISHAAYA, I.; DE COCK, A.; DEGHEELE, D. Pyriproxyfen, a potent supressor of egg hatch and adult formation of the greenhouse whitefly (Homoptera: Aleyrodidae). Journal of Economic Entomology, v.87, p.1185-1189, 1994

LIU, T-X.; STANSLY, P.A. Effects of pyriproxifen on three species of Encarsia (Hymenoptera: Aphelinidae), endoparasitoids of Bemisia argentifolii (Homoptera: Aleyrodidae). Journal of Economic Entomology, v.90, p.404-411, 1997.

LOURENÇÃO, A.L.; NAGAI, H. Surtos populacionais de Bemisia tabaci no Estado de São Paulo. Bragantia, v.53, p.53-59, 1994.

VILLAS BÔAS, G.L.; FRANÇA, F.H.; ÁVILA, A.C.de; BEZERRA, I.C. Manejo integrado da mosca branca Bemisia argentifolii. Brasília: EMBRAPA, CNPH, 1997. 11p. (Circular Técnica, 9).

Recebido em 21.11.00 